\title{
Unilateral spatial neglect due to right frontal lobe haematoma
}

Shinichiro Maeshima, Kazuyoshi Funahashi, Mitsuhiro Ogura, Toru Itakura, Norihiko Komai

\begin{abstract}
Two patients with unilateral spatial neglect caused by right frontal lobe lesions underwent cerebral blood flow studies. A 54-year-old, right-handed woman developed left hemiplegia and frontal lobe neglect associated with cerebral haemorrhage after surgical excision of a frontal tumour. A 66-year-old, righthanded woman developed a haemorrhage in the right frontal lobe caused by rupture of an aneurysm. This was followed by left hemiplegia and frontal lobe neglect. In both cases, ${ }^{123}$ I-iodoamphetamine single photon emission CT disclosed a reduction in regional cerebral blood flow localised along the circumference of the haematoma in the frontal lobe, but did not reveal any lesions in the parieto-occipital junction. These findings suggest that, in these two cases, the frontal lobe neglect was caused by lesions confined to the frontal lobe.
\end{abstract}

(F Neurol Neurosurg Psychiatry 1994;57:89-93)

Unilateral spatial neglect is a symptom wherein the patient disregards objects existing in the outer half. Lesions in the parietooccipital junction in the right hemisphere have generally been considered responsible for this symptom. ${ }^{12}$ Unilateral spatial neglect is also thought to reflect a disturbance in the spatial distribution of directed attention. ${ }^{3}$ There have been reports of this symptom occurring in association with lesions in the occipital and frontal lobes, subcortical lesions involving the thalamus or putamen, and left hemispheric lesions. ${ }^{4-10}$ The phenomenon of unilateral spatial neglect reflects deficits in at least one of the centres responsible for spatial processing, selective attention, mental representation, awareness, or premotor planning. The responsible lesion and mechanism are still controversial. We report on two cases of frontal lobe neglect associated with right frontal lobe damage. We discuss the cerebrovascular kinetics and lesions responsible in these cases.

\section{Case reports}

CASE 1

A 54-year-old, right-handed woman had headache and vomiting in the morning of July 13, 1990. Her precontrast CT scan on July 16 showed a slight abnormality. She was
Department of Neurological Surgery, Wakayama Medical College, Wakayama, Japan

S Maeshima

K Funahashi

M Ogura

T Itakura

N Komai

Correspondence to:

Dr Shinichiro Maeshima, Department of Neurological Surgery, Wakayama Medical College, 27 Nanaban-cho, Wakayama 640, Japan.

Received 17 August 1992 and in revised form 9 March 1993.

Accepted 31 March 1993

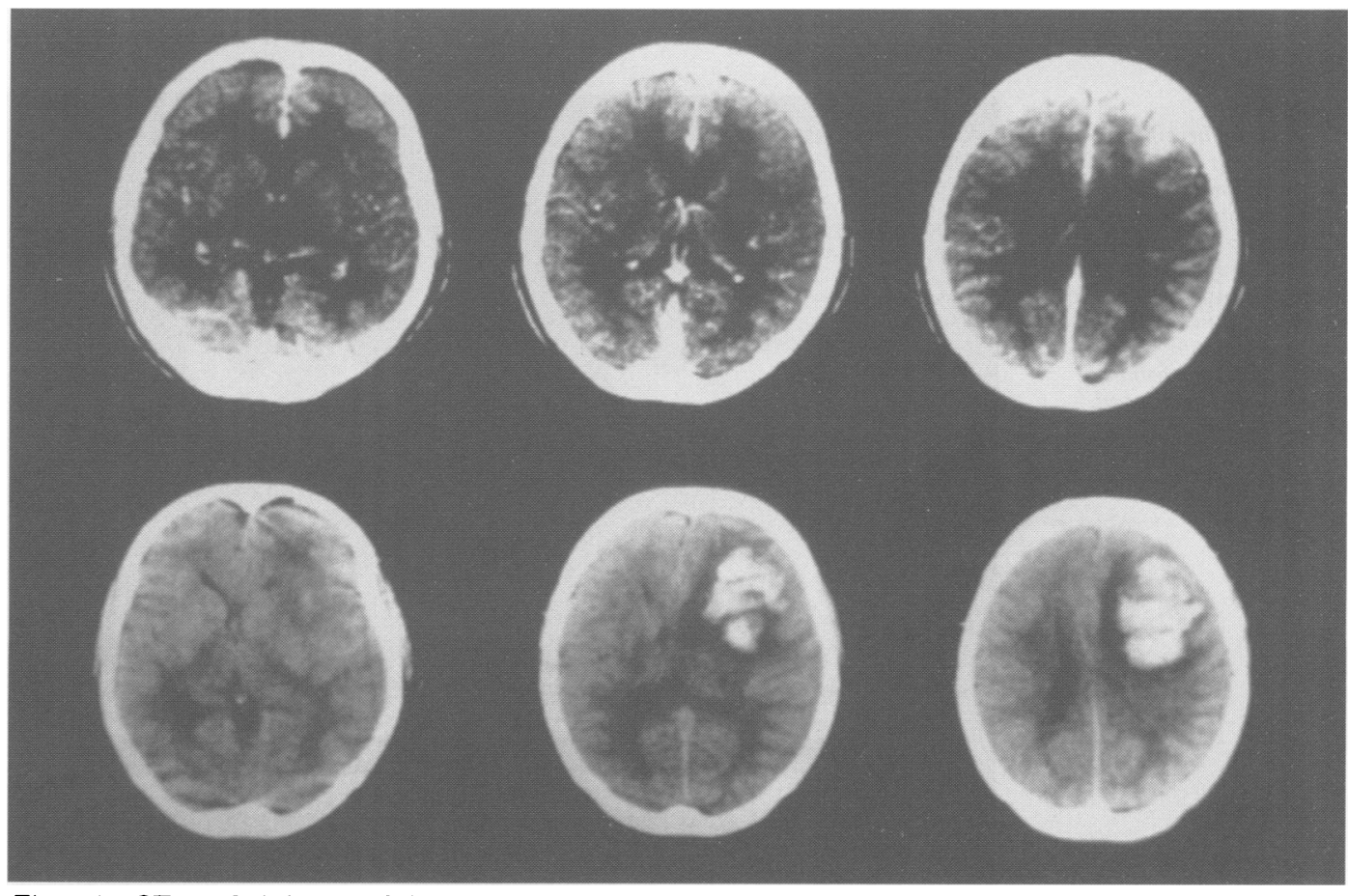

Figure 1 CT on admission revealed an enhanced tumour in the right frontal lobe (top row). CT on the third day after surgery revealed a haematoma confined to the right frontal lobe (bottom row). 
Figure 2 Neglect of the left side was seen in some neuropsychological tasks.

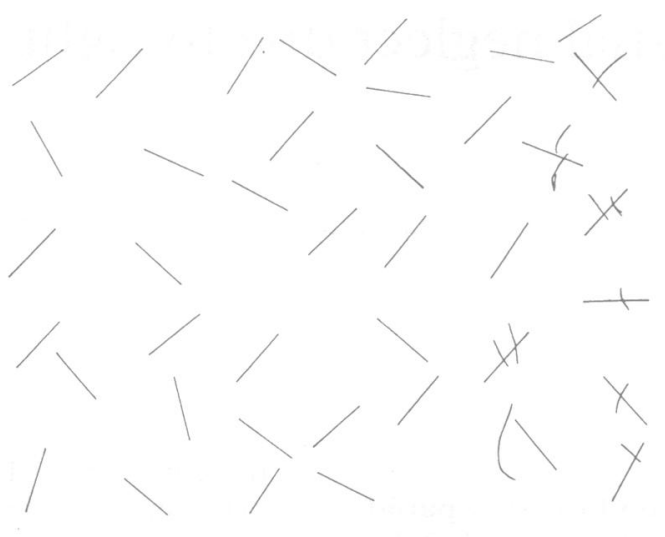

referred to our hospital for close examination on July 19. On admission she was alert, oriented, and appropriately motivated. Cranial nerves were intact. There were neither sensory nor motor deficits. A CT scan revealed a contrast enhancing tumour in the right frontal lobe (fig 1, top); cerebral angiography showed tumour stain and vessels feeding from the right middle meningial artery. These findings supported a diagnosis of a convexity meningioma in the right frontal lobe. On August 20, frontal craniotomy and total meningioma excision were performed under general anaesthesia. After the operation, the patient regained consciousness and was without sensory or motor deficit. On the third postoperative day, however, a disturbance in consciousness and a left hemiplegia were noted. A CT scan revealed a high density area confined to the right frontal lobe (fig 1 , bottom). A postoperative intracerebral haematoma was surgically removed on August 23. The day after the evacuation, she became alert and oriented. She had full visual fields by a confrontational testing, normal extraocular movements, left-sided facial palsy and a severe left hemiparesis. Perception of pin, touch, vibration, proprioception, graphaesthesia, and stereognosis were normal.

\section{Neuropsychological examination}

On September 2, the 10th postoperative day, neurological examinations were performed. Dementia was not observed and Hasegawa's simple intelligence test scale was $27 \cdot 0 / 32 \cdot 5$ (subnormal). Digit span was performed successfully for up to seven digits, and word fluency (animal names) was 13 per minute. Aphasia was not seen and there were no abnormalities in reading vertically written letters and words, or in writing her own name. Neglect of the left side was seen in line bisection, line cancellation, figure copying tests, and in describing scenic pictures (fig 2).
Figure 3

${ }^{123}$ I-iodoamphetamine single photon emission $C T$ was performed in the second week after surgery. It showed a decrease in regional cerebral blood flow in the circumference of the haematoma in the frontal lobe in early images (top row) and a redistribution phenomenon in delayed images (bottom row).

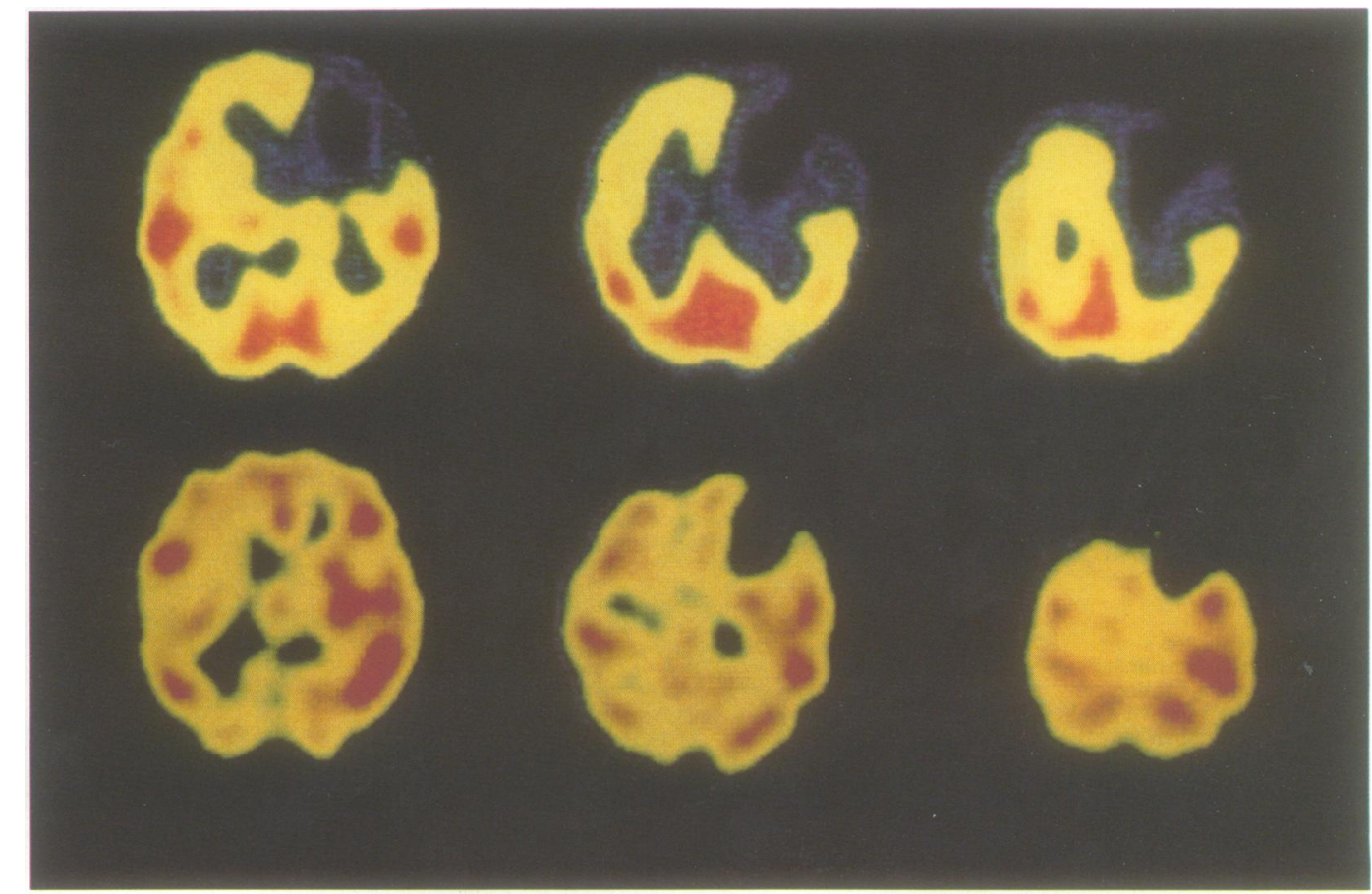


Figure $4 C T$ scan on the day after the onset of headache and vomiting in patient 2 disclosed

haemorrhage in the right frontal lobe (top row).

Postoperative CT revealed a low density area in the lateral side of the right

frontal lobe (bottom row).

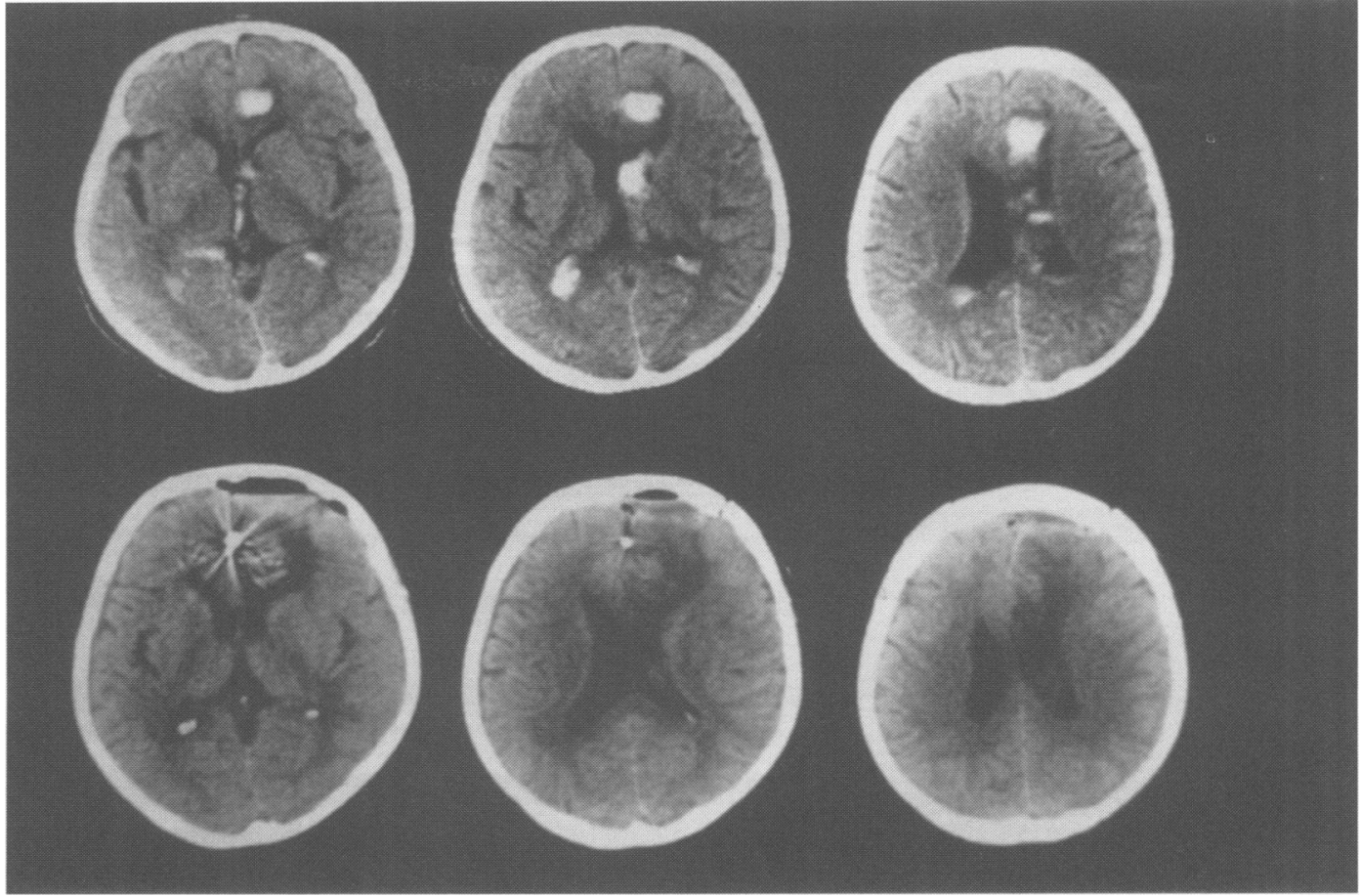

There was left sided extinction in the visual, auditory, and tactile modalities on bilateral, simultaneous stimulation. Ideomotor apraxia, ideational apraxia, or anosognosia were not observed.

\section{Clinical course}

A ${ }^{123}$ I-iodoamphetamine single photon emission CT (SPECT) study was performed in the second postoperative week. It showed a decrease in the regional cerebral blood flow along the circumference of the haematoma in the frontal lobe in early images and a redistribution phenomenon in the delayed images (fig 3). A Wechsler adult intelligence scale verbal IQ was 92 . Several neuropsychological examinations were performed on September 30 (the 38th postoperative day), and there was some improvement in the left-sided neglect. Abnormalities were still noted in line bisection, line cancellation tests, and extinction in all sensory modalities on bilateral, simultaneous stimulation. Subsequently, leftside neglect gradually improved and disappeared three months later. Repeat ${ }^{123}$ I-IMP SPECT showed that the reduced regional cerebral blood flow in the frontal lobe remained unchanged.

\section{CASE 2}

A 66-year-old, right-handed woman had an attack of headache and vomiting on February 7,
Figure 5

${ }^{123}$ I-iodoamphetamine single photon emission $C T$ in the third week after surgery showed a decrease in regional cerebral blood flow in the circumference of the haematoma in the

frontal lobe in early images (top row) and a

redistribution phenomenon in delayed images (bottom row).

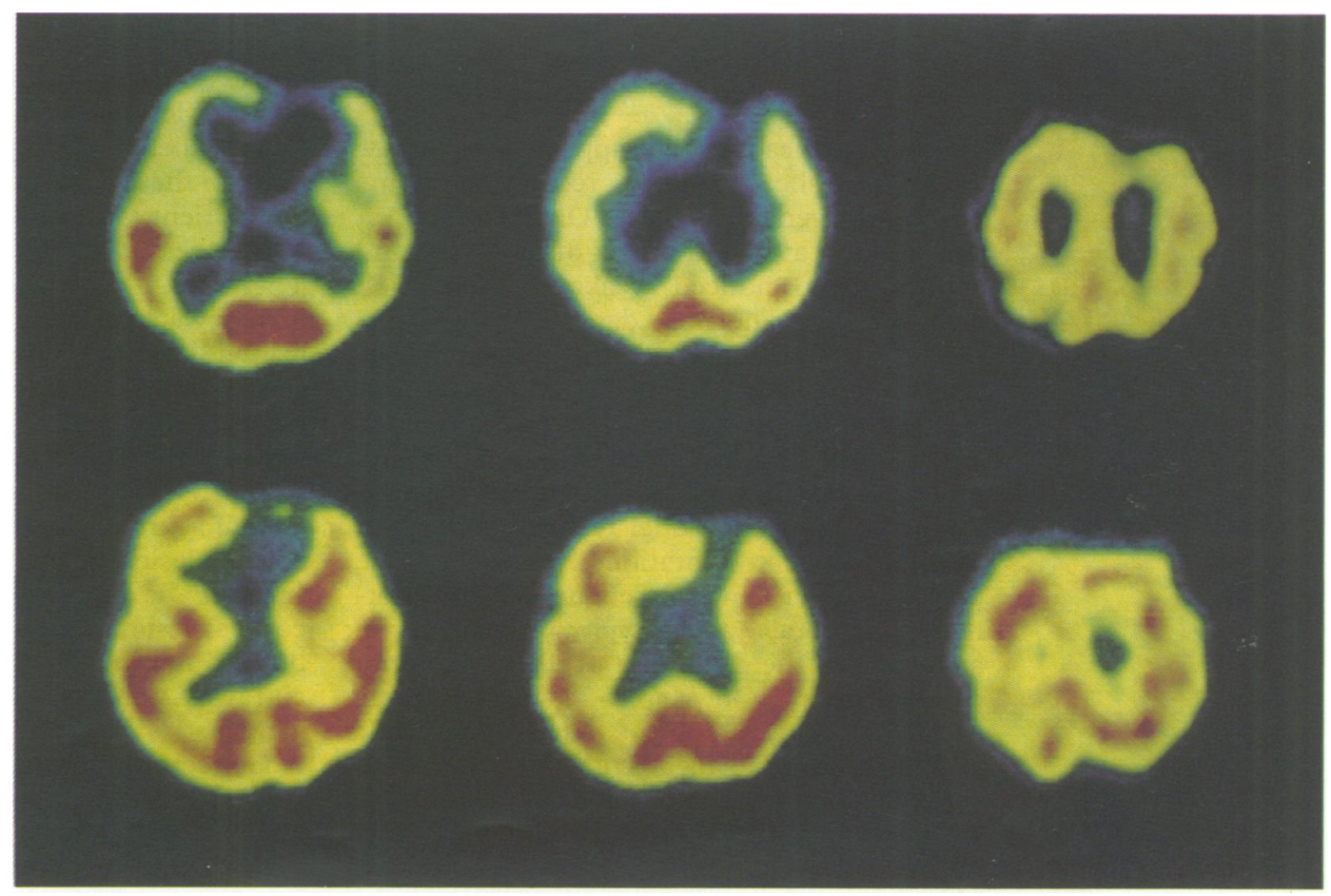


Figure 6 Neglect of the left side was seen in some neuropsychological tasks.
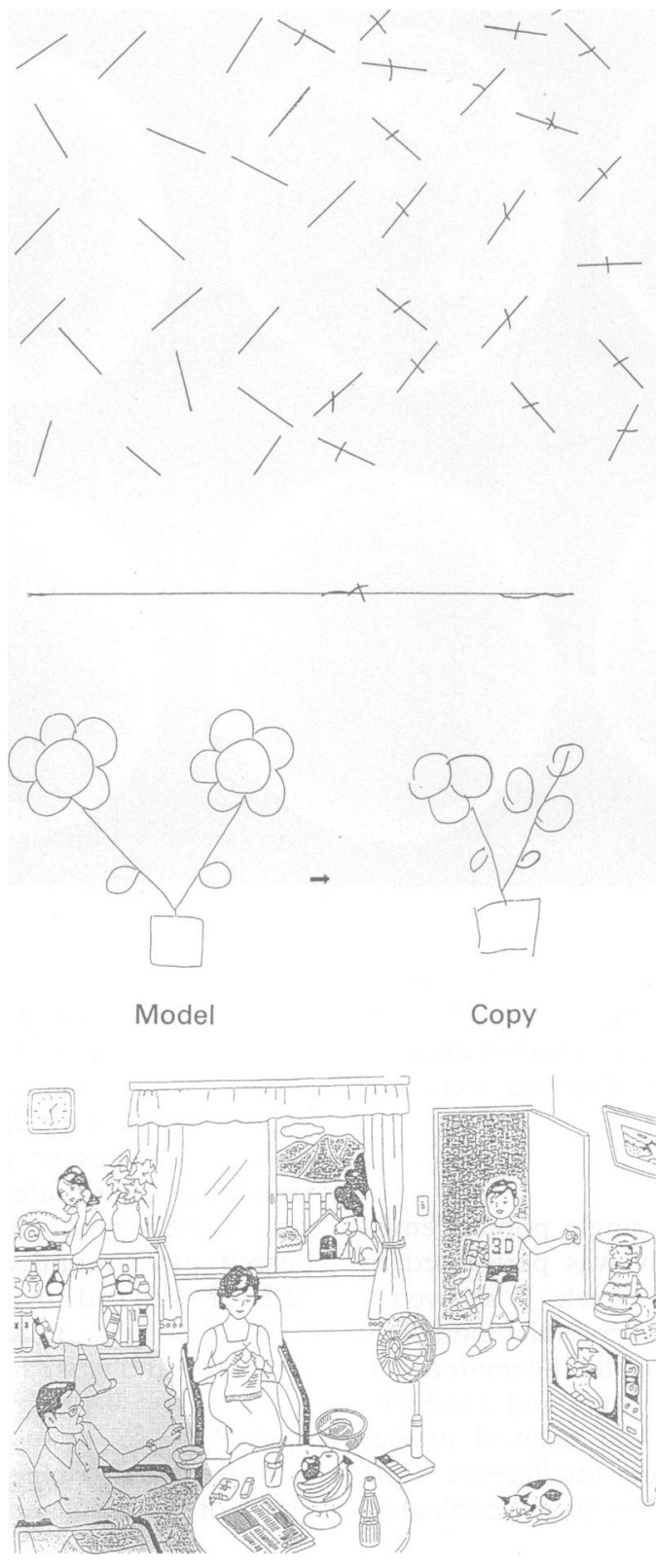

1991. The headache disappeared in a few days, but she remained resting at home because of general malaise. On February 19, she suffered a second attack of headache and vomiting and noticed weakness of her left arm and leg. The next day a CT scan disclosed a haemorrhage in the right frontal lobe (fig 4, top). On March 11, when she was referred to our hospital, she was alert and oriented. She had left-sided facial palsy and moderate left hemiparesis but no visual field defect by confrontational testing. Perception of pin, touch, vibration, proprioception, graphaesthesia, and stereognosis were normal. Cerebral angiography revealed an aneurysm in the distal part of the left anterior cerebral artery. On March 22, neck clipping and total removal of the haematoma were successfully performed with an interhemispheric approach. Neurological symptoms, such as hemiplegia, did not obviously improve.

\section{Neuropsychological examination}

On April 6, the second postoperative week, neurological examinations were carried out. The patient had mild disorientation and memory disturbance but no general dementia or aphasia. Digit span was performed successfully up to six digits, and word fluency (animal names) was two per minute. There were no abnormalities in reading vertically written letters and words or in writing her own name. Left-sided neglect, however, was observed in line bisection, line cancellation, and figure copying tests, and in describing scenic pictures (fig 6). There was left-sided extinction in the visual, auditory, and tactile modalities on bilateral, simultaneous stimulation. Ideomotor apraxia, ideational apraxia, or anosognosia were not observed.

\section{Clinical course}

Postoperative CT revealed a low density area in the lateral aspect of the right frontal lobe (fig 4, bottom). A ${ }^{123}$ I-IMP SPECT scan in the third postoperative week showed a decrease in regional cerebral blood flow along the circumference of the haematoma in the frontal lobe in early images and a redistribution phenomenon in the delayed images (fig 5). In the fourth postoperative week, the patient was referred to another hospital for rehabilitation.

\section{Discussion}

Heilman et $a l^{4}$ reported six cases with unilateral spatial neglect caused by lesions in the frontal lobe, naming the syndrome "frontal lobe neglect". However, their study was made before the introduction of the CT scan, so there may be ambiguity in the identification of lesion sites, except in the one autopsied case. Damasio et $a^{5}$ reported five cases of frontal lobe neglect; two of them had lesions in the basal ganglia and the other three in the left frontal lobe. Employing CT, Kubo ${ }^{6}$ investigated the causative lesions in 28 cases of unilateral spatial neglect, and found that none of these lesions were confined to the frontal lobe. Valler et $a l^{7}$ reported that lesions confined to the frontal lobe were detected in only one patient with unilateral spatial neglect, of 110 cases with right hemispheric stroke. Therefore, it may well be that cases of frontal lobe neglect caused by lesions confined to the frontal lobe are rare. Imamura et $a l^{8}$ have stated some conditions for unilateral spatial neglect associated with frontal lobe lesions: firstly, the lesion extends to the basal ganglia; secondly the lesion involves the occipital lobe as well as the frontal lobe; or thirdly, the subcortical lesion in the frontal lobe is extensive. In both the present cases, an extensive subcortical lesion was noted in the frontal lobe but did not involve the parieto-occipital lobe, and was not easily detectable in the basal ganglia.

Concerning the mechanism of frontal lobe neglect caused by subcortical lesions, Heilman et $a l^{1}$ have hypothesised that pressure on the surrounding tissues by the lesion 
produces a disconnection of nerve fibres projecting to the cortex, or damages cortical function at the subcortical level. Valler et al ${ }^{9}$ examined functional lesions in two patients with unilateral spatial neglect and subcortical vascular lesions using SPECT, and suggested that the right frontotemporoparietal cortex could be the site of the lesion responsible. We have examined functional lesions in thalamic neglect using xenon-enhanced CT and hypothesise that the origin of the disturbed cortical function can be found in the parietooccipital lobe. ${ }^{10}$ In the present cases, however, we did not find a reduction in cerebral blood flow in the parieto-occipital junction, even at the time when frontal lobe neglect was being observed. Therefore, neglect would appear to occur in association with a single lesion confined to the frontal lobe. In case 1 , when frontal lobe neglect had already disappeared, SPECT showed no improvement in the reduction of cerebral blood flow, but rather a redistribution phenomenon in the delayed image. This phenomenon seemed to be due to activation of the remaining cortex.

Heilman et $a l^{14}$ emphasised the participation of Brodmann's areas eight, nine and 46, including a dorsolateral part of the frontal lobe, in frontal lobe neglect. They have suggested that this condition could occur because of an insult to the connecting fibres, including fibres conveying visual, somatic, and auditory information, between the frontal cortex and the inferior parietal lobule. They have also reported that the supplementary motor area and cingulate gyrus, both of which are connected to a dorsolateral portion of the frontal lobe, are responsible in frontal lobe neglect. ${ }^{4}$ Mesulam et al ${ }^{311}$ suggested that four cerebral regions - the posterior, parietal, frontal, and reticular components-provide an integrated network for the modulation of attention within extrapersonal space. Each component region has a unique functional role that reflects its profile of anatomical connectivity, and each gives rise to a different clinical type of unilateral spatial neglect when damaged. Lesions in only one component of this network yield parietal unilateral spatial neglect, whereas those that encompass all the components result in profound deficits that transcend the mass effect of the larger lesion. Illustratively, it is thought that a right frontal injury causes only left-sided, exploratory motor hemispatial neglect. ${ }^{12}$ From the present cases, it appears that an extensive subcortical lesion in the frontal lobe involved the connecting fibres between the frontal cortex and the inferior parietal lobule, resulting in frontal lobe neglect. The study of attention and movement in macaque monkey by Rizzolatti et $a l^{13}$ reported that area six caused neglect in somatosensory and visual modalities but area eight did not cause somatosensory deficit.

In this study, we could not determine whether the patients had exploratory motor hemispatial neglect, because patients were not challenged with an exploratory motor task. But unilateral spatial neglect in these two patients was most obvious during the cancellation, figure copying, and picture scene tests. Additionally, there was left-sided extinction in the visual, auditory, and tactile modalities on bilateral simultaneous stimulation. The network for directed attention is so heavily interconnected, and the lesion in the frontal lobe was so large, that the unilateral spatial neglect in the two patients appears to be multifactorial. There was no qualitative difference in the performance of the two women, but the unilateral spatial neglect in patient 1 was more severe than that of patient 2 . It seems that this difference in severity was dependent on the volume of the haematoma in the right frontal lobe.

In our two cases of frontal lobe neglect caused by lesions confined to the right frontal lobe, the lesion did not involve the parietooccipital lobe, even on cerebral blood flow studies. This finding suggests that frontal lobe neglect in these two cases was caused by a mechanism that does not involve the parieto-occipital lobe or thalamus.

1 Heilman KM, Valenstein E, Watson RT. Localization of neglect. In Kertesz A, ed. Localization in neuropsychology. New York: Academic Press, 1983:471-92.

2 Kubo K. Localization of unilateral spatial neglect. Higher Brain Funct Res 1989;9:106-11.

3 Mesulam MM, Attention, confusional states, and negiect. In Mesulam MM, ed. Principles of behavioral neurology. Philadelphia: FA Davis, 1985:125-68.

4 Heilmann MH, Valenstein E. Frontal lobe neglect in man Neurology 1971;22:660-4.

5 Damasio AR, Damasio H, Chui HC. Neglect following damage to frontal lobe or basal ganglia. Neuropsychologia 1980;18:123-32.

6 Kubo K. Unilateral spatial neglect. Adv Neurol (fpn) 1980;24:598-609.

7 Vallar G. Perani D. The anatomy of unilateral neglect after right hemisphere stroke lesions. A clinical/CT-scan after right hemisphere stroke lesions. A clinical/CT-scan 493-501.

8 Imamura $Y$, Uemura $K$. Unilateral spatial neglect following damage to right frontal lobe. Higher Brain Funct Res (Fpn) 1989;9:211-8.

9 Vallar G, Perani D, Cappa SF, et al. Recovery from aphasia and neglect after subcortical stroke: neuropsychological and cerebral perfusion study. $\mathcal{F}$ Neurol Neurosurg Pyychiatry 1988;51:1269-76.

10 Maeshima S, Hyoutani G, Terada $T$, Nakamura $Y$, Yokote H, Komai N, et al. Effect of thalamic neglect on activities of daily living. Sogo Rehabil 1990;18:445-50.

11 Daffner KR, Ahern GL, Weintraub S, Mesulam MM Dissociated neglect behavior following sequential strokes in the right hemisphere. Ann Neurol 1990;28: 97-101.

12 Liu GT, Bolton AK, Price BH, Weintraub S. Dissociated perceptual-sensory and exploratory-motor neglect.

13 Rizzolatti G, Matelli M, Pavesi G. Deficits in attention and movement following the removal of postarcuate (area 6) and prearcuate (area 8) cortex in macaque (area 6) and prearcuate (area 8)
monkeys. Brain 1983;106:655-73. 